for $i=0,1,2, \cdots$. We found that, for $-13, \leq i \leq 13$,

$$
\left\|e\left(x^{\prime}\right)\right\|^{2}=450.10^{-8}, \quad\left\|e\left(y^{\prime}\right)\right\|^{2}=450.10^{-8}, \quad\left\|e\left(f^{\prime}\right)\right\|^{2}=386.10^{-8} .
$$

In roughing up $x^{\prime}, y^{\prime}, f^{\prime}$ at $i=-10$, as well as in smoothing these quantities, the values had been left unchanged at zero, in order to avoid absurd entries there.

The smoothing again did diminish the error vectors somewhat:

$$
\left\|e\left(x^{*}\right)\right\|^{2}=383.10^{-8}, \quad\left\|e\left(y^{*}\right)\right\|^{2}=379.10^{-8}, \quad\left\|e\left(f^{*}\right)\right\|^{2}=341.10^{-8} \text {. }
$$

On the other hand, $\|e(g)\|^{2}=370.10^{-8}$. Further, $\|e(h)\|^{2}=99.10^{-8}$, while $\left\|e\left(f^{*}\right)\right\|^{2}=$ $128.10^{-8}$ for the corresponding entries between $i=-11$ and $i=11$. For the derivatives the following results were obtained:

$$
\|e(\psi)\|^{2}=118.10^{-8}, \quad\|e(\varphi)\|^{2}=161.10^{-8}, \quad\left\|e\left(F^{*}\right)\right\|^{2}=132.10^{-8} .
$$

5. Conclusion. In view of the results obtained above the following conclusions, at least for the examples discussed, seem to be justified:

(a) $f^{*}$ is not significantly better than $g$;

(b) the improvement $h$ due to the further smoothing of $f^{*}$ may be worthwhile;

(c) $F^{*}$ is about as good as $\psi$, while $\varphi$ is considerably worse than either of the foregoing. In summary, then, it may be stated that if functional values $f$ alone are desired, possibility I of Sec. 2 would seem to be adequate. However, if rates of change $d f / d t$ also are of interest, then possibility II should be utilized.

\title{
REFERENCE
}

1. See, for example, Whittaker-Robinson, The calculus of observations, 4th ed., 1944, p. 291

\section{TRANSVERSE VELOCITIES IN FULLY DEVELOPED FLOWS*}

\author{
By STEPHEN H. MASLEN
}

\section{By STEPHEN H. MASLEN (Lewis Flight Propulsion Laboratory, Cleveland, Ohio)}

We consider an incompressible flow in a channel whose generators are parallel and lie in the $x$-direction. The driving force, either a pressure gradient or some kind of a body force, is also in the $x$-direction. If the driving force and the flow velocities are independent of $x$, then the flow is said to be fully developed. In such a case, it is usually assumed that the velocity components normal to the $x$-direction vanish identically. However, in some cases it is not clear that this must be true. One such example is that of a free convective flow where the velocity ( $x$-direction) shows a cellular structure [1], being sometimes in one direction (up, say) and sometimes in the other (down). It is the purpose of the present note to show that regardless of these circumstances the transverse velocity componeuts in a fully developed incompressible flow must vanish everywhere.

Consider a fully developed incompressible flow, where the velocity components $u, v, w$, are in the (cartesian) $x, y, z$ directions. If $P, \rho, \nu$, and $F$ are pressure, density, 
kinematic viscosity, and body force, respectively, the equations become

$$
\left.\begin{array}{c}
v_{\nu}+w_{z}=0 \\
v u_{y}+w u_{z}+P_{x} / \rho=\nu \Delta u+F \\
v v_{y}+w v_{z}+P_{y} / \rho=\nu \Delta v \\
v w_{y}+w w_{z}+P_{z} / \rho=\nu \Delta w
\end{array}\right\}
$$

where subscripts denote partial differentiation and $\Delta$ is the Laplacian operator. The boundary conditions are that $u=v=w=0$ at the walls.

The first of Eqs. (1) implies the existence of a stream function $\psi$, such that $v=\psi_{2}$, $w=-\psi_{y}$. Using this definition, the last two of Eqs. (1) can be combined to read

$$
L(\psi) \equiv \nu \Delta \Delta \psi+\left(\psi_{y} \Delta \psi_{z}-\psi_{z} \Delta \psi_{\nu}\right)=0 .
$$

The appropriate boundary conditions are that $\psi_{y}=\psi_{z}=0$ at the wall. One can observe that

$$
\begin{aligned}
\psi L(\psi)-\nu(\Delta \psi)^{2}= & \left\{\psi\left(\nu \Delta \psi_{z}+\psi_{\nu} \psi_{z z}-\psi_{z} \psi_{\nu z}\right)-\nu \psi_{z} \Delta \psi-\frac{1}{2} \psi_{\nu}\left(\psi_{\nu}^{2}+\psi_{z}^{2}\right)\right\}_{z} \\
& +\left\{\psi\left(\nu \Delta \psi_{\nu}-\psi_{z} \psi_{\nu \nu}+\psi_{\nu} \psi_{\nu z}\right)-\nu \psi_{\nu} \Delta \psi+\frac{1}{2} \psi_{z}\left(\psi_{\nu}^{2}+\psi_{z}^{2}\right)\right\}_{\nu} \\
\equiv & \left\{\frac{\psi P_{\nu}}{\rho}-\nu \psi_{z} \Delta \psi-\frac{1}{2} \psi_{\nu}\left(\psi_{\nu}^{2}+\psi_{z}^{2}\right)\right\}_{z} \\
& +\left\{-\frac{\psi P_{z}}{\rho}-\nu \psi_{\nu} \Delta \psi+\frac{1}{2} \psi_{\nu}\left(\psi_{\nu}^{2}+\psi_{z}^{2}\right)\right\}_{\nu}
\end{aligned}
$$

If this equation is integrated over the cross section, $D$, of the channel, there follows, using Green's theorem

$$
\begin{aligned}
\int_{D}\left[\psi L(\psi)-\nu(\Delta \psi)^{2}\right] d y d z=\int_{C}\left[-\frac{\psi P_{\psi}}{\rho}+\nu \psi_{z} \Delta \psi+\frac{1}{2} \psi_{\nu}\left(\psi_{\nu}^{2}+\psi_{z}^{2}\right)\right] d y \\
+\left[-\frac{\psi P_{z}}{\rho}-\nu \psi_{\nu} \Delta \psi+\frac{1}{2} \psi_{z}\left(\psi_{\nu}^{2}+\psi_{z}^{2}\right)\right] d z,
\end{aligned}
$$

where $C$ is the boundary. If Eqs. (2) are noted, this becomes simply

$$
\nu \int_{D}(\Delta \psi)^{2} d y d z=+\frac{1}{\rho} \int_{C} \psi\left(P_{\nu} d y+P_{s} d z\right)=+\frac{1}{\rho} \int_{C} \psi P . d s,
$$

where $s$ is the distance along the boundary in the $y-z$ plane.

In general the cross section, $D$, is not simply connected, but has a boundary consisting of several closed curves $c_{i}$. Because $\psi_{x}, \psi_{\nu}$ vanish on each, it follows that $\psi$ is a constant, $\psi_{i}$, on each. Then

$$
\nu \int_{D}(\Delta \psi)^{2} d y d z=\frac{1}{\rho} \sum_{i} \psi_{i} \int_{c_{i}} P, d s
$$

Because the pressure must be single valued, the right hand integrals must vanish. Hence, so does the left hand one. This is only possible if $\Delta \psi=0$ everywhere. Then, subject to the boundary conditions of Eqs. (2), $\psi$ can be at most a constant and the transverse velocities must vanish everywhere. 
Hence, in fully developed incompressible flows, there can be no transverse velocity components. The flow is then found from the second of Eqs. (1), dropping the inertia terms. Of course, for free convective flow (for example, Ref. [2]), an energy equation must also be included.

\section{REFERENCES}

1. D. P. Timo, Free convection in narrow vertical sodium annuli, Knolls Atomic Power Laboratory; Report No. KAPL-1082, March 1954

2. S. Ostrach, Combined natural and forced-convection laminar flow and heat transfer of fluids with and without heat sources in channels with linearly varying wall temperatures, NACA, TN 3141, 1954

\section{ON AN INEQUALITY OF LIAPOUNOFF*}

\section{BY AUREL WINTNER (The Johns Hopkins University)}

1. Let $\boldsymbol{p}(t)$ be a continuous function which is positive on the $t$-interval under consideration. Instead of $p(t)>0$, it will be sufficient to assume that $p(t) \geqq 0$, provided that $p(t)>0$ holds on a dense $t$-set. The role of this proviso will be that of excluding the existence of a function $x(t)$ which satisfies the differential equation

$$
x^{\prime \prime}+p(t) x=0
$$

and is a non-ranishing constant on some $t$-interval. If such a solution $x(t)$ of $(1)$ is disregarded [and (1) cannot have two, linearly independent, such solutions in any case], then, besides the continuity of $p(t)$, only the assumption $p(t) \geqq 0$ will be needed. Only real-ralued solutions $x(t)$ will be considered, and the trivial solution, $x(t) \equiv 0$, will be excluded.

It is clear from (1) that $x^{\prime \prime}(t) \geqq 0$ or $x^{\prime \prime}(t) \leqq 0$ at a given $t$ according as $x(t)<0$ or $x(t)>0$ at that $t$. Hence the graph of $x=x(t)$ must turn its concavity toward the $t$-axis at every $t$. Since the clustering of zeros of the derivative $x^{\prime}(t)$ has been excluded, it follows that the zeros of $x(t)$ separate, and are separated by, the zeros of $x^{\prime}(t)$ [provided that either $x(t)$ or $x^{\prime}(t)$ has at least two zeros]. Let a closed $t$-interval [c,d] be called a primitive interval of $x(t)$ if neither $x(t)$ nor $x^{\prime}(t)$ has any zero in the interior of $[c, d]$. Such an interval $[c, d]$ will be called a complete primitive interval of $x(t)$ if for no $\epsilon>0$ is $[c-\epsilon, d+\epsilon]$ a primitive interval of $x(t)$, that is, if $x(t) \neq 0$ and $x^{\prime}(t) \neq 0$ for $c<t<d$ but either $x(c)=0$ and $x^{\prime}(d)=0$ or $x^{\prime}(c)=0$ and $x(d)=0$. Note that $x^{\prime}(c) \neq 0$ and $x(d) \neq 0$ in the first case, and that $x(c) \neq 0$ and $x^{\prime}(d) \neq 0$ in the second case, since the simultaneous vanishing of $x(t)$ and $x^{\prime}(t)$ leads to the trivial solution.

2. The purpose of this note is to show that, owing to the concept of a primitive interval, a theorem of Liapounoff (see below) can be extended from his "disconjugate" case to the general case, as follows:

If $p(t)$ is continuous and non-negative on a closed t-interval $[a, b]$, and if $[a, b]$ consists of exactly $n$ primitive intervals of some solution $x(t)$ of $(1)$, then

$$
\int_{a}^{b} p(t) d t>n^{2} /(b-a)
$$

*Received March 28, 1957. 